\title{
QUALITY OF LIFE OF PATIENTS UNDERGOING SURGICAL TREATMENT OF LOWER LIMB CANCER
}

\author{
Maria Iakovleva $^{1}$, Olga Shchelkova $^{1}$, \& Ekaterina Usmanova ${ }^{2}$ \\ ${ }^{1}$ Department of Medical Psychology and Psychophysiology, Saint Petersburg State University (Russia) \\ ${ }^{2}$ Department of Medical Rehabilitation, National Medical Research Center of Oncology \\ named after N.N. Blokhin (Russia)
}

\begin{abstract}
Patients suffering from oncological diseases are the focus of attention of both physicians and psychologists. Although tumor lesions of bones and soft tissues are a relatively rare condition, its effect on the person' mind and lifestyle are significant, and its treatment is a challenge, also in terms of patient's adaptation to the disease and therapy. There are various strategies for treating this pathology; all of them are associated with high-tech medicine focused on maintaining or improving patients' quality of life (QoL).

The aim of the present research is to study the psychological characteristics and QoL of patients undergoing surgical treatment of tumor lesions of bones and soft tissues of lower limbs.

Material and methods. 36 patients were examined (mean age 58,22; 19 - men). The SF-36 questionnaire, Ways of Coping Questionnaire (WCQ), and Big Five Personality Test (BFI) were used. Treatment by means of the isolated limb perfusion technique was prescribed to 15 patients (group 1); 21 patients were subjected to lower limb amputation due to their disease (group 2).

Results. It was found that patients who underwent amputation are characterized by lower rates on the coping scale 'accepting responsibility' than patients from the perfusion group $(p<0.05)$; at the same time, patients from the second group had higher values on the 'openness' scale of the BFI compared to the first group $(p<0.1)$.

The study of the relationship between patients' QoL parameters, personality and coping showed that in the first group the values on the coping scale 'seeking social support' negatively correlate with 'bodily pain' $(p<0.01)$, and 'escape-avoidance' negatively correlates with 'social functioning' $(p<0.01)$. In the second group, the following significant correlations between personality traits and QoL were revealed: 'extraversion' is positively associated with 'physical functioning', 'role-emotional' and 'mental health' $(p<0.01)$, 'agreeableness' has a negative correlation with 'bodily pain' and 'general health' $(p<0.01)$, 'neuroticism' is negatively related with 'bodily pain' and 'general health' ( $p<0.01)$, 'openness' is positively associated with 'bodily pain' and 'general health' $(p<0.01)$.

Conclusion. The data obtained emphasizes the importance of taking into account personality characteristics in the management of patients with cancer, including tumor lesions of the bones and soft tissues, as well as the dynamic nature of QoL and its close interconnection with the stage and strategy of treatment and patients' personality. Psychological support for patients is required for their successful adaptation to the illness and therapy.
\end{abstract}

Acknowledgement. This research was supported by the Russian Foundation for Basic Research (RFBR) (Grant No 20-013-00573).

Keywords: Health related quality of life, coping, cancer, surgical treatment.

\section{Introduction}

Tumor lesions of bones and soft tissues of limbs (such as sarcoma and some others) are not considered the most common. However, the risk of death and disability for patients is quite high, and its therapy is challenging and presents specific difficulties. There are various approaches to treating this cohort of patients, including radiation, chemotherapy, surgical treatment; in any case, limb salvage treatment is a priority (Crago \& Lee, 2016). However, it is not always possible to achieve recovery using the mentioned methods, and there is a need of limb amputation. Isolated limb perfusion (ILP) is an alternative to amputation; it has been explored for more than 50 years (Martin-Tellez et al., 2020). The 
procedure is considered safe and is recommended even for elderly patients (van Etten et al., 2003), but it is not used very often.

The benefits of such treatment are numerous; the technique is less traumatic, patients' functioning after surgery gets compromised to a minimum, it induces a high tumor response rate and leads to a high limb salvage rate. Thus, the application of this method is justified. There is also evidence of a significantly worse physical functioning of patients after amputation in comparison with patients after ILP, as well as higher rates of anxiety; these differences are especially pronounced when amputations are performed at the above-knee level (Pardasaney et al., 2006).

Despite the proven efficacy of ILP, there is also evidence of a high recurrence rate after ILP (Bhangu et al., 2013). The described contradictions are fundamental in the issue of determining a treatment strategy for patients with tumor lesions of bones and soft tissues of limbs. In modern-day medicine, one of the key factors that is taken into account when choosing therapy is the psychological factor - patient's health-related quality of life (QoL), which includes numerous aspects of physical, mental and social functioning. Researchers hypothesize a higher QoL in patients who have undergone ILP compared to patients who have undergone amputation (Mason et al., 2013). However, the problem was acknowledged as controversial and complex for evaluating (Chang et al., 1989). The available data on the QoL of patients after surgery are contradictory: despite the incomparably lower trauma of ILP, the posterior level of QoL is not higher than after amputation.

The aim of the present research is to study the psychological characteristics and QoL of patients undergoing surgical treatment of tumor lesions of bones and soft tissues of limbs.

\section{Methods}

The study was performed at the N.N. Blokhin National Medical Research Center of Oncology (Moscow, Russia). 36 patients undergoing treatment for tumor lesions of bones and soft tissues of lower limbs were examined; the mean age of the sample was 58.22 years old, $52.78 \%$ (19 patients) were male. Treatment by means of the isolated limb perfusion technique was prescribed to 15 patients (group 1); 21 patients were subjected to lower limb amputation due to their disease (group 2).

The psychological investigation included the SF-36 Questionnaire ("The Short Form (36) Health Survey") (SF-36) to assess patients' QoL and its parameters, the Ways of Coping Questionnaire (WCQ) to assess patients' coping behaviour, and the Big Five Personality Inventory (BFI) to assess patients' personality traits.

\section{Results}

The study of patients' QoL by means of the SF-36 Questionnaire showed no significant differences between the groups in terms of their QoL parameters.

Analysis of patients' data obtained through the WCQ and the BFI allowed to reveal the following differences in the psychological characteristics of the groups (see Table 1). It was found that patients who underwent amputation (group 2) are characterized by lower rates on the coping scale Accepting responsibility of the WCQ than patients from the perfusion group $(p<0.05)$, meaning they are less prone to accept and acknowledge their role in the emergence of a problem and less willing to take responsibility for its solution. At the same time, patients from the second group tended to have higher values on the Openness scale of the BFI compared to the first group $(p<0.1)$.

Table 1. Differences in psychological characteristics of patients undergoing ILP/amputation.

\begin{tabular}{|l|c|c|c|}
\hline Patient characteristic & $\begin{array}{c}\text { Group 1 (ILP) } \\
\text { N=15 (M) }\end{array}$ & $\begin{array}{c}\text { Group 2 (amputation) } \\
\text { N=21 (M) }\end{array}$ & $p$-value \\
\hline Openness (BFI) & 26.18 & 31.10 & $<.1$ \\
\hline Accepting responsibility (WCQ) & 51.64 & 44.67 & $<.05$ \\
\hline
\end{tabular}

Note. ILP - Isolated limb perfusion; M - mean value; BFI - Big Five Personality Inventory; WCQ - Ways of Coping Questionnaire.

The study of the relationship between patients' QoL parameters, personality and coping allowed to establish the following. No significant correlations were revealed between QoL and personality traits in patients who underwent ILP. Meanwhile, several correlations were revealed between QoL parameters and coping strategies in the first group (see Table 2): Role-Physical Functioning has a correlation with the Self-controlling coping $(p<0.05)$; Social Functioning is negatively related to the Confrontive $(p<0.05)$, Distancing $(p<0.05)$, and Escape-Avoidance $(p<0.01)$ copings; Bodily Pain negatively correlates with the copings Seeking social support $(p<0.01)$ and Positive reappraisal $(p<0.05)$. 
Table 2. Correlations between QoL parameters (SF-36 Questionnaire) and coping strategies (Ways of Coping Questionnaire) in patients undergoing ILP.

\begin{tabular}{|c|c|c|c|c|c|c|c|c|}
\hline \multirow{2}{*}{$\begin{array}{l}\text { Scales of the Ways of } \\
\text { Coping Questionnaire }\end{array}$} & \multicolumn{8}{|c|}{ SF-36 scales } \\
\hline & $\mathrm{GH}$ & $\mathrm{PF}$ & RP & $\mathrm{RE}$ & $\mathrm{SF}$ & $\mathrm{BP}$ & VT & $\mathrm{MH}$ \\
\hline Confrontive & & & & & $-.709 *$ & & & \\
\hline Distancing & & & & & $-.825^{*}$ & & & \\
\hline Self-controlling & & & $.812^{*}$ & & & & & \\
\hline Seeking social support & & & & & & $-.854 * *$ & & \\
\hline Accepting responsibility & & & & & & & & \\
\hline Escape-Avoidance & & & & & $-.882 * *$ & & & \\
\hline Planful problem-solving & & & & & & & & \\
\hline Positive reappraisal & & & & & & $-.733 *$ & & \\
\hline
\end{tabular}

Note. ${ }^{*} p<.05 ;{ }^{* *} p<.01$. GH - General Health; PF - Physical Functioning; RP - Role-Physical Functioning; RE - Role-Emotional; SF - Social Functioning; BP - Bodily Pain; VT - Vitality; MH - Mental Health.

In the second group, the following significant correlations between personality traits and QoL were revealed: Extraversion is positively associated with Physical Functioning, Role-Emotional Functioning and Mental Health $(p<0.01)$, Agreeableness has a negative correlation with Bodily Pain and General Health $(p<0.01)$, Neuroticism is negatively related with Bodily Pain and General Health $(p<0.01)$, Openness is positively associated with Bodily Pain and General Health $(p<0.01)$.

\section{Discussion}

Studies available to date do not provide a definite answer on the level and dynamics of QoL of patients undergoing ILP. Thus, there is evidence of a higher QoL of these patients compared to the same age population average values, among those who had not been diagnosed with cancer (Noorda, 2004). The authors attribute their results to a life reevaluation of patients who faced a vital threat and experienced repeated recurrences and surgery. On the other hand, there are opposite data showing lower QoL of patients compared with a healthy sample (Eiser et al., 2001).

Another contrariety that arises in the analysis of this topic is the discrepancy in the data regarding the effect of the performed surgery type on the later QoL of patients: the field presents both data on significantly higher QoL of patients after ILP compared to amputations (e.g., Mason et al., 2013), and data on the absence of significant differences in QoL of these two groups of patients, up to the conclusion that there is no need to take this factor into account when choosing a therapy strategy (Zahlten-Hinguranage et al., 2004). Our results partly accord with these findings; but we cannot agree that patients' QoL is not related to the characteristics of the treatment and should not be taken into account when considering different treatment strategies.

The traumatic effect of surgery on patients' psychological status seems apparent; thus, symptoms of psychological distress to the extent of PTSD were revealed on a sample of patients suffering from tumor lesions of the extremities and undergoing ILP (Thijssens et al., 2006); however, not all researchers confirm similar changes in the patients' psychological status after surgery.

In summary, the conclusion that was drawn more than 35 years ago on the ambiguousness of psychological outcomes of limb-salvage surgery compared to amputation (Weddington, Seagraves, \& Simon, 1985), remains relevant and requires further detailed research.

\section{Conclusions}

The research on the QoL of patients undergoing surgical treatment of tumor lesions of bones and soft tissues and their psychological characteristics will be continued. It is fundamental to take into account the time of the surgery, its complications and concomitant diseases, the social status of patients, along with the use of more precise tools to assess the QoL. The data obtained also emphasizes the importance of taking into account personality characteristics in the management of patients with cancer, as well as the dynamic nature of QoL and its close interconnection with the stage and strategy of treatment and patients' personality. Psychological support for patients is required for their successful adaptation to the illness and therapy. 


\section{Acknowledgements}

This research was supported by the Russian Foundation for Basic Research (RFBR) (Grant No 20-013-00573).

\section{References}

Bhangu, A., Broom, L., Nepogodiev, D., Gourevitch, D., \& Desai, A. (2013). Outcomes of isolated limb perfusion in the treatment of extremity soft tissue sarcoma: a systematic review. European Journal of Surgical Oncology, 39(4), 311-319. https://doi.org/10.1016/j.ejso.2012.12.018

Chang, A. E., Steinberg, S. M., Culnane, M., Lampert, M. H., Reggia, A. J., Simpson, C. G., Hicks, J. E., White, D. E., Yang, J. J., \& Glatstein, E. (1989). Functional and psychosocial effects of multimodality limb-sparing therapy in patients with soft tissue sarcomas. Journal of clinical oncology: official journal of the American Society of Clinical Oncology, 7(9), 1217-1228. https://doi.org/10.1200/JCO.1989.7.9.1217

Crago, A. M., \& Lee, A. Y. (2016). Multimodality Management of Soft Tissue Tumors in the Extremity. The Surgical clinics of North America, 96(5), 977-992. https://doi.org/10.1016/j.suc.2016.05.001

Eiser, C., Darlington, A. S., Stride, C. B., \& Grimer, R. (2001). Quality of life implications as a consequence of surgery: limb salvage, primary and secondary amputation. Sarcoma, 5(4), 189-195. https://doi.org/10.1080/13577140120099173

Martin-Tellez, K. S., van Houdt, W. J., van Coevorden, F., Colombo, C., \& Fiore, M. (2020). Isolated limb perfusion for soft tissue sarcoma: Current practices and future directions. A survey of experts and a review of literature. Cancer Treatment Reviews, 88, 102058. https://doi.org/10.1016/j.ctrv.2020.102058.

Mason, G. E., Aung, L., Gall, S., Meyers, P. A., Butler, R., Krüg, S., Kim, M., Healey, J. H., \& Gorlick, R. (2013). Quality of life following amputation or limb preservation in patients with lower extremity bone sarcoma. Frontiers in oncology, 3, 210. https://doi.org/10.3389/fonc.2013.00210

Noorda, E.M. (2004). Indications and Results of Isolated Limb Perfusion (UvA Dissertations). Amsterdam: Amsterdam University Press.

Pardasaney, P. K., Sullivan, P. E., Portney, L. G., \& Mankin, H. J. (2006). Advantage of limb salvage over amputation for proximal lower extremity tumors. Clinical orthopaedics and related research, 444, 201-208. https://doi.org/10.1097/01.blo.0000195413.16150.bc

Thijssens, K. M., Hoekstra-Weebers, J. E., van Ginkel, R. J., \& Hoekstra, H. J. (2006). Quality of life after hyperthermic isolated limb perfusion for locally advanced extremity soft tissue sarcoma. Annals of surgical oncology, 13(6), 864-871. https://doi.org/10.1245/ASO.2006.05.023

van Etten, B., van Geel, A. N., de Wilt, J. H. W., \& Eggermont, A. M. M. (2003). Fifty tumor necrosis factor-based isolated limb perfusions for limb salvage in patients older than 75 years with limb-threatening soft tissue sarcomas and other extremity tumors. Annals of Surgical Oncology, 10, 32-37. https://doi.org/10.1245/ASO.2003.03.076

Weddington, W. W., Jr, Segraves, K. B., \& Simon, M. A. (1985). Psychological outcome of extremity sarcoma survivors undergoing amputation or limb salvage. Journal of clinical oncology: official journal of the American Society of Clinical Oncology, 3(10), 1393-1399. https://doi.org/10.1200/JCO.1985.3.10.1393

Zahlten-Hinguranage, A., Bernd, L., Ewerbeck, V., \& Sabo, D. (2004). Equal quality of life after limb-sparing or ablative surgery for lower extremity sarcomas. British journal of cancer, 91(6), 1012-1014. https://doi.org/10.1038/sj.bjc.6602104 